\title{
Especificaciones del servicio de medición y control del riesgo vascular
}

\author{
Elaborado por \\ Comisión de Servicios Profesionales Farmacéuticos de SEFAC \\ Revisado por \\ Vicente J. Baixauli, Emilio García Jiménez, Ana Molinero \\ Grupo de trabajo de Hipertensión arterial y riesgo vascular de SEFAC
}

\section{PALABRAS CLAVE}

Riesgo cardiovascular, hipertensión arterial, medida de la presión arterial, farmacia comunitaria, farmacéutico comunitario, servicios profesionales farmacéuticos, retribución

\section{ABREVIATURAS}

AMPA: automedida de la presión arterial

AVAC: años de vida ajustados a calidad GIAF-UGR: Grupo de Investigación en Atención Farmacéutica de la Universidad de Granada. HTA: hipertensión arterial IRC: insuficiencia renal crónica LOPD: Ley Orgánica de Protección de Datos

MAFC: medida aislada en farmacia comunitaria

MAPA: medida ambulatoria de la presión arterial

NICE: The National Institute for Health and Care Excellence

PA: presión arterial

PNT: procedimiento normalizado de trabajo

RCV: riesgo cardiovascular

RUM: revisión de uso de la medicación SEFAC: Sociedad Española de Farmacia Familiar y Comunitaria.

SEH-LELHA: Sociedad Española de Hipertensión-Liga Española para la Lucha contra la Hipertensión SPD: sistema personalizado de dosificación

SPF: servicios profesionales

farmacéuticos

ZAP: zona de atención personalizada

\section{KEYWORDS}

Cardiovascular risk, high blood pressure, measurement of blood pressure, community pharmacy, community pharmacist, professional pharmaceutical services, remuneration

\section{RESUMEN}

En abril de 2013, la Sociedad Española de Farmacia Familiar y Comunitaria (SEFAC) presentó su propuesta sobre servicios profesionales farmacéuticos (SPF), cuyo fin es cubrir las necesidades relacionadas tanto con la atención de los pacientes que utilizan medicamentos como con la salud pública. Esta propuesta ofrece un planteamiento sobre la implantación y desarrollo de los SPF con el objetivo de impulsar su prestación por las farmacias comunitarias en los próximos años. De acuerdo con dicha propuesta, todos los SPF que constituyen el catálogo de servicios contarán con un documento de especificaciones.

El objeto de este documento de especificaciones es definir y caracterizar el servicio de prevención y control del riesgo vascular con un doble objeto:

- Apoyar al farmacéutico comunitario y a sus colaboradores en el ofrecimiento, prestación, difusión, financiación y concertación de este servicio.

- Servir de guía a los farmacéuticos comunitarios que desean implantar este servicio en la farmacia o elaborar un procedimiento normalizado de trabajo para su realización.

Este documento se complementa con la Guía de actuación para el farmacéutico comunitario en pacientes con hipertensión arterial (HTA) y riesgo cardiovascular (RCV). Documento de consenso GIAF-UGR, SEFAC y SEH-LELHA y con el programa impacHta: formación SEFAC y SEH-LELHA en hipertensión y riesgo vascular. Se recomienda también el uso de las tablas para el cálculo del riesgo vascular Framingham, SCORE (Systematic Coronary Risk Evaluation) y Regicor (Registre Gironí del Cor).

Specifications from the vascular risk measuring and control service

\section{ABSTRACT}

In April 2013, the Sociedad Española de Farmacia Familiar y Comunitaria (SEFAC) [Spanish Society for Family and Community Pharmacy] submitted its proposal regarding professional pharmaceutical services (PPS), whose aim is to cover healthcare for patients who use medicines and public health. This proposal offers an approach regarding the implementation and development of PPSs to boost their provision for community pharmacies in the coming years. According to this proposal, all the PPSs included in the services catalogue will be given a specifications document.

The purpose of this specifications document is to define and characterize the vascular risk prevention and control service with a double purpose:

- Support community pharmacies and their collaborators in the offering, providing, distributing, funding and coordinating of this service.

- Guide community pharmacies that want to implement this service at the pharmacy or prepare a standardized work procedure.

This documented is supplemented by the Guidelines for community pharmacies for patients with high blood pressure (hypertension) and cardiovascular risk (CVR). Consensus document GIAF-UGR, SEFAC and SEH-LELHA and with the impacHta program: SEFAC and SEH-LELHA training for high blood pressure and cardiovascular risk. The use of the Framingham, SCORE (Systematic Coronary Risk Evaluation) and Regicor (Registre Gironí del Cor) tables are also recommended to calculate cardiovascular risk.

Conflicto de intereses: Los autores declaran no existir conflicto de intereses en relación con el contenido del documento.

Cite este artículo como: Comisión Servicios Profesionales Farmacéuticos SEFAC. Especificaciones del servicio de medición y control del riesgo vascular. Farmacéuticos Comunitarios. 2016 Sep 30; 8(3):18-22. doi:10.5672/FC.2173-9218. (2016/Nol8).003.04 


\section{Introducción y justificación}

En abril de 2013, la Sociedad Española de Farmacia Familiar y Comunitaria (SEFAC) presentó su propuesta sobre servicios profesionales farmacéuticos (SPF) [1], cuyo fin es cubrir las necesidades relacionadas tanto con la atención de los pacientes que utilizan medicamentos, como con la salud pública. Esta propuesta ofrece un planteamiento sobre la implantación y desarrollo de los SPF con el objetivo de impulsar su prestación por las farmacias comunitarias en los próximos años. De acuerdo con dicha propuesta todos los SPF que constituyen el catálogo de servicios contarán con un documento de especificaciones.

Las guías publicadas por las sociedades europeas de Hipertensión Arterial y de Cardiología sobre el manejo de la hipertensión arterial (HTA) se centraban en los valores de presión arterial (PA) como la única variable para determinar la necesidad y el tipo de tratamiento. Posteriormente, estas mismas Sociedades desarrollaron conjuntamente una serie de recomendaciones sobre la prevención de la enfermedad coronaria en la práctica clínica, destacando la necesidad de cuantificar el riesgo cardiovascular total $[2,3]$.

El abordaje de la salud de un paciente o usuario de una farmacia en los aspectos relacionados con el riesgo de padecer una enfermedad vascular en un plazo determinado, es decir, el riesgo de manifestarse una enfermedad a nivel coronario (angina, infarto agudo de miocardio), a nivel cerebrovascular (ictus, accidente isquémico transitorio) o a nivel periférico (arteriopatía periférica), puede realizarse utilizando herramientas que ayudan a predecir esta probabilidad, como las tablas para el cálculo del riesgo vascular Framingham [4], SCORE (Systematic Coronary Risk Evaluation) [5] y REGICOR (Registre Gironi del Cor) [6,7].

\section{Definición \\ y descripción breve}

Este servicio consiste en la medida y el control del riesgo vascular (RV) del paciente, dentro de la farmacia comunitaria. La medida del RV puede realizarse desde la farmacia comunitaria, a través de distintos méto- dos de medición validados en función de las características y necesidades del paciente, mientras que el control se realiza a través del seguimiento de los valores obtenidos respecto de los objetivos de control de ese paciente, el asesoramiento farmacéutico al paciente y la colaboración con el resto de profesionales sanitarios.

El método de medida se basa en el cálculo del RV con las tablas SCORE para países de bajo RV [5]. Para su cálculo es necesario conocer cierta información clínica relacionada con algunos factores de RV del paciente usuario de este servicio.

Esta información es la siguiente:

- Resultado analítico de una glucemia basal y/o de la hemoglobina glicosilada o diagnóstico de diabetes (con el objetivo de saber si el paciente es diabético o no).

- Valores de presión arterial sistólica obtenidas mediante AMPA.

- Resultado analítico del colesterol total en sangre.

- Si el paciente es fumador o no.

- La edad y sexo.

\section{Objetivos}

Objetivos generales

- Determinar el riesgo vascular (RV) de los pacientes y usuarios.

- Asesorar e implicar a los pacientes en el control de su RV.

\section{Objetivos específicos}

- Mejorar el conocimiento del paciente sobre los estilos de vida saludables, medidas higiénico-dietéticas y farmacoterapéuticas relacionadas con los factores de RV: hipertensión, dislipemia, diabetes, tabaquismo, obesidad, estrés, etc.

- Informar de los resultados obtenidos a los pacientes y a los profesionales sanitarios que los precisen.

- Colaborar con el médico u otros profesionales de la salud que atiendan al paciente en el control de su RV.

\section{Población/ pacientes diana}

La población diana como tal podría ser cualquier paciente o usuario que acude a la farmacia, que presente al menos un factor de RV: hipertensión, diabetes, dislipemia, tabaquismo, obesidad, estrés, y/o tenga una edad mayor a 40 años en hombres y 50 años en mujeres.

Sin embargo, los pacientes diana para recibir este servicio están condicionados por su edad, debido a que para el cálculo del RV se utilizan unas tablas en función de ésta.

\section{Recursos necesarios}

\section{Recursos materiales}

1. Zona de atención personalizada (ZAP)

En el servicio de medición y control del riesgo vascular se requiere una relación entre el farmacéutico y el paciente. La confianza y la confidencialidad son pilares básicos, por lo que es imprescindible disponer de una zona diferenciada y distinta de la zona de dispensación que garantice la intimidad (paciente y el farmacéutico puedan hablar sin ser escuchados por cualquier otra persona, incluyendo el personal que trabaja en la farmacia) y la relajación del paciente.

2. Materiales impresos

a. Cartelería (opcional).

b. Folletos educativos para los pacientes.

c. Hojas de registro del RV, derivación al médico, etc.

\section{Documentación y registros}

a. Ficha de cada paciente, (consentimiento informado, registros, datos de medicación...).

$b$. Informes dirigidos al médico.

Se registrarán los datos relativos a las enfermedades de RV diagnosticadas, datos de control de los factores de RV (presión arterial, perfil lipídico, glucemia) y a los tratamientos utilizados en su control.

Es necesario disponer de un sistema de registro seguro de toda la información y documentación utilizada, preferiblemente un ordenador con el software adecuado e impresora. Se recomienda disponer de ellos en formato digital para su mejor cumplimentación, archivo y recuperación.

A la hora de complementar la información clínica del paciente hay que distinguir entre la información procedente de los registros de dispensación u otros servicios de la propia farmacia de aquella procedente de otros profesionales sanitarios o es proporcionada por el paciente (informes médicos, pruebas analíticas, informes de alta hospitalaria, etc.). 
4. Fuentes de información sobre gestión de riesgo vascular y tratamientos.

Es necesario disponer de la Guía Europea sobre prevención de la Enfermedad Cardiovascular en práctica clínica [2], así como de las actualizaciones que cada año promueven todas las sociedades científicas a nivel nacional e internacional [8].

Asimismo, es conveniente disponer de acceso a fuentes de información sobre medicamentos, como la base de datos del consejo general de colegios oficiales de farmacéuticos.

\section{Utillaje}

- Tabla SCORE para países de bajo RV.

En caso de no disponer de los valores de los factores de RV necesarios:

- Tensiómetro clínicamente validado para la medición de la PA.

- Dispositivo/s de obtención de valores analíticos de factores de RV.

\section{Recursos humanos}

Los recursos humanos son el factor determinante a la hora de desarrollar la medición y control del riesgo vascular, dado que se trata de un servicio profesional donde la preparación y el tiempo de los profesionales resultan imprescindibles. Por ello, todos los farmacéuticos que presten este servicio deberán estar capacitados específicamente.

Responsable del servicio: con el fin de organizar mejor este servicio es conveniente que exista un farmacéutico responsable del servicio. Cuando en la farmacia sólo trabaja un farmacéutico éste será el responsable del mismo. Si trabaja más de un farmacéutico se debe nombrar un responsable entre todos los farmacéuticos del equipo. La elección del farmacéutico responsable del servicio se debería tomar en función de las habilidades de comunicación, la actitud frente al servicio y la experiencia asistencial. El responsable del servicio, junto con el farmacéutico titular, debería marcar los objetivos e indicadores del servicio, con el fin de evaluarlos cada año junto a la satisfacción de los pacientes.

Continuidad del servicio: para facilitar la continuidad del servicio a lo largo del tiempo es aconsejable que todos los farmacéuticos de la farmacia estén capacitados para poder prestarlo, así se evita su paralización ante periodos de vacaciones, bajas por enfermedad, o ausencia del responsable de la farmacia.

Obligaciones del farmacéutico responsable del servicio: el farmacéutico responsable debe asumir ciertas tareas específicas relacionadas con la provisión del servicio.

Personal técnico: el personal técnico debidamente adiestrado por el farmacéutico podrá auxiliar al farmacéutico responsable en diversas tareas adecuadas a su formación.

\section{Recursos temporales}

La medición y control del riesgo vascular es una actividad clínica que obliga a entrevistar al paciente, determinar el RV, así como tomar decisiones respecto al paciente que utiliza medicamentos, y realizar los registros correspondientes de la actividad, lo que supone un consumo de tiempo.

\section{Derivación}

En el transcurso del servicio, el farmacéutico puede detectar situaciones que excedan los objetivos del mismo y precisen de una intervención profesional adicional. En estos casos, el farmacéutico indicará al paciente la conveniencia de derivarle a otro servicio, en el que se aborde la situación detectada.

La derivación quedará reflejada en el informe del servicio, así como en el procedimiento normalizado de trabajo (PNT) del servicio, donde se especificarán las situaciones y circunstancias que la determinen. La derivación puede ser de dos tipos: interna y externa.

\section{Derivación interna}

La situación detectada puede tratarse mediante otro servicio disponible en la misma farmacia comunitaria, sea por el mismo profesional $\mathrm{u}$ otro farmacéutico del establecimiento. Se registrará la situación, el servicio interno al que se deriva y la justificación de la misma.

Los casos más habituales de derivación interna en este servicio pueden ser:

- Ausencia de datos sobre los factores de RV necesarios para realizar el cálculo del RV, requiriéndose el Servicio de medición y control de PA, el Servicio de seguimiento y control de otros parámetros bioquímicos (glucemia, colesterol, etc.), u otro.

- Detección de hábitos higiénico-dietéticos inadecuados: puede necesitarse un servicio de Educación para la salud, de asesoramiento nutricional y dietético, de cesación tabáquica.

- Problemas con la farmacoterapia: requerirá la puesta en marcha de un servicio de revisión farmacoterapéutica e incluso de seguimiento farmacoterapéutico.

- Problemas en la adherencia al tratamiento o dificultad en la utilización de los medicamentos: precisará de un servicio de RUM, SPD, formulación magistral...

\section{Derivación externa}

La situación detectada no puede tratarse en la propia farmacia, por no prestar dicho servicio, exceder las competencias del personal farmacéutico de dicha farmacia, o requerir de otro nivel asistencial por lo que es preciso derivar a otro profesional sanitario. Igualmente se realizará un parte de derivación, previo consentimiento del paciente, adjuntando toda la información necesaria para el otro profesional.

El caso más habitual de derivación externa en este servicio es:

- Problemas farmacoterapéuticos, que afecten directamente al tratamiento y precisen ser tratados por el médico responsable del paciente.

\section{Protocolización}

El servicio de medición y control del riesgo vascular deberá estar protocolizado y el PNT deberá ser seguido y conocido por todo el equipo de la farmacia.

El PNT es una herramienta para el profesional, por lo que debe adaptarse a la situación concreta y específica de los profesionales que ejercen en dicha farmacia. Por tanto, sería conveniente que los farmacéuticos de cada farmacia desarrollasen su propio PNT, como por ejemplo partiendo de PNT estándares [2] y amoldándolos a la situación particular.

Asimismo, los informes que se generen deberán seguir unas pautas de rigor y claridad a fin de ofrecer la máxima información posible. 


\section{Documentación y registros}

La documentación del servicio de medición y control del riesgo vascular está constituida principalmente por el consentimiento informado para la prestación del servicio, que incluirá el consentimiento para el tratamiento de datos personales de acuerdo con la LOPD.

Es necesario pedir autorización firmada al paciente. Una vez firmada la autorización por el farmacéutico y por el paciente, se le entregará una copia de este documento. Si un paciente se niega a dar su consentimiento, el farmacéutico no debe prestarle el servicio.

Normalmente en el mismo consentimiento se incluye la posibilidad de proporcionar la autorización del paciente para que el farmacéutico comparta información sobre la intervención con médicos u otros proveedores de atención médica, cuando ésta sea necesaria.

El registro de los datos obtenidos permiten realizar un informe que será evaluado por el médico que tomará las acciones oportunas. En función de ello $\mathrm{y}$ realizando un nuevo control posteriormente, siempre que hubiese cambios en la medicación (tipo de medicamento, dosis o pauta), se obtendrán unos resultados que permitan demostrar el impacto de la actuación farmacéutica en el paciente. El registro podrá realizarse en soporte papel o preferiblemente en soporte informático garantizando la confidencialidad y seguridad de los datos, adaptándose a la LOPD.

Los registros necesarios para el servicio deben contener fundamentalmente:

- Información personal del paciente: nombre y apellidos, datos de contacto, etc.

- Información sanitaria del paciente: tratamiento farmacológico relacionado con el riesgo vascular y problemas de salud relevantes.

- Información sobre la medida y el control del RV, junto con problemas detectados y medidas recomendadas si procede

- Información sobre la derivación, si precisa, donde se indique el problema detectado y el servicio y al profesional al que se deriva.

\section{Calidad, certificación y mejora continua}

Con el fin de impulsar y garantizar la calidad de este servicio, SEFAC recomienda la inclusión de este servicio en un programa de aseguramiento de la calidad.

El requisito de la certificación tiene como objetivo atestiguar -a través de la emisión de un documento o certificado- que un servicio, persona o empresa se ajusta a unas normas técnicas de calidad determinadas.

Hay que distinguir entre tres tipos de certificaciones:

1. Certificación de profesionales: los farmacéuticos comunitarios que realicen el servicio de medición y control del riesgo vascular deberán contar con la certificación de la capacitación correspondiente.

2. Certificación de establecimientos y equipos: las farmacias comunitarias que presten el servicio de medición y control del riesgo vascular deberán disponer de la certificación de que se dispone de las instalaciones y los recursos materiales necesarios para su prestación.

3. Certificación y validación de PNT: los PNT utilizados en la realización del servicio de medición y control del riesgo vascular deben haber sido validados para su utilización en la práctica clínica.

SEFAC ofrecerá además a los farmacéuticos interesados participar en un programa de supervisión externa de la calidad con el fin de poder compararse con otras farmacias y poder mejorar este servicio.

Independientemente de ello, se recomienda realizar periódicamente auditorías internas del servicio y encuestas de satisfacción a los pacientes y a los profesionales que lo prestan.

\section{Resultados obtenidos}

En España hay diferentes trabajos que han analizado el beneficio de proveer a la población de intervenciones en salud que mejoren y bajan el riesgo vascular desde la farmacia comunitaria. En este sentido, algunas experiencias de intervención a pacientes con riesgo vascular moderado/alto desde la farmacia, ya sea con educación sanitaria básica o seguimiento farmacoterapéutico, han demostrado su efectividad y/o eficacia en mejorar el riesgo vascular de la población objeto de estos trabajos tras un periodo de entre 6-12 meses de intervención [9-12].

\section{Retribución y en su caso financiación}

Retribución

La realización de este servicio en la farmacia comunitaria conlleva una serie de costes que requieren ser cubiertos para que este servicio pueda garantizar su viabilidad en el tiempo. Por tanto, la forma de retribución establecida para dicho servicio debe al menos cubrir los costes que ocasiona su realización. Hay que tener en cuenta que la realización de este servicio podría suponer al farmacéutico comunitario una gran inversión económica por diferentes motivos como la necesidad de un cambio en la estructura de la farmacia con la creación de zonas de atención personalizada, la utilización de otros aparatos, un mayor número de nuevas herramientas informáticas, el entrenamiento del personal o incluso contratar más personal. Por otro lado supone un consumo adicional de tiempo para la realización de este servicio (tiempo para la toma de datos con el paciente, estudio, toma de decisiones, diversos registros, etc.) con lo que esto supone de un mayor coste. En definitiva, para establecer la retribución de este servicio hay que determinar en primer lugar los costes y posteriormente el beneficio que se pretende obtener.

Los costes requeridos para su prestación incluyen:

Costes fijos (no varían con el número de pacientes atendidos):

Incluyen los costes proporcionales derivados de la amortización de las instalaciones y en su caso del aparataje que se ha de adquirir para la realización del servicio. En definitiva:

- Las inversiones realizadas para realizar este servicio (instalaciones, equipamiento y su mantenimiento): ZAP, ordenador, impresora...

- Los costes proporcionales de los gastos generales de la farmacia (luz, limpieza, acceso a internet, software, seguros, aplicación LOPD, etc.) y de la capacitación del personal.

- Cinta métrica. Coste aproximado de $1 €$.

- Tablas SCORE actualizadas para países de bajo RV.

En caso de que el paciente no conociera los valores analíticos de los factores de RV necesarios para el cálculo del RV, sería necesario disponer de: 
- Tensiómetros (con los diferentes manguitos disponibles, según perímetro del brazo del paciente). El coste de adquisición del aparataje (clínicamente validado) para AMPA oscila sobre 60-100 € en el año 2013, en función del modelo y marca seleccionado. Baterías de los tensiómetros, archivo y la documentación necesaria, así como la calibración de los aparatos anualmente, los gastos de seguros de responsabilidad civil y de los aparatos.

- Dispositivo para el seguimiento y control de parámetros bioquímicos. $\mathrm{Al}$ objeto de obtener el dato de colesterol total, necesario para el cálculo del RV (incluyendo guantes de vinilo, algodón, tiras reactivas, lancetas, capilares...). El precio de estos dispositivos es variado dependiendo de la marca, tipo y número de pruebas que pueda realizar oscilando entre 600 y $5.000 €$.

Costes variables o gastos de funcionamiento del servicio: incluyen los costes del material fungible (papel, tinta, folletos, cartelería, hojas para registros, tiras reactivas para la determinación de los valores de los factores de RV si procede, etc).

Coste del farmacéutico que el realiza el servicio y depende del tiempo empleado.

La entrevista y el cálculo del RV según el método SCORE pueden requerir alrededor de 10 minutos. En caso de que además fuera necesario obtener los valores de los factores de RV para el cálculo del RV (presión arterial, prueba de colesterol total, PA...) este tiempo podría incrementarse en unos 10-15 minutos más.

El coste económico relacionado con el tiempo empleado por el farmacéutico se ha calculado en base al coste por hora de un farmacéutico, que puede variar según el convenio actual vigente al que esté sujeto la oficina de farmacia, entre 16,97 y 22,52 euros/hora.

\section{Financiación}

El pago de este servicio, al igual que el resto de los SPF puede conjugar diferentes modalidades:

- pago íntegro por el paciente

- cobertura total por sanidad pública/seguro privado
- copago del paciente con cobertura parcial de la sanidad pública/seguro privado.

Dados los costes que supone la aparición de un accidente vascular por el desconocimiento del RV, su influencia en numerosas enfermedades y los resultados obtenidos, estaría justificado que fuese financiado total o parcialmente, de forma selectiva para determinados pacientes y fuera incluido en la cartera de servicios de las entidades proveedoras de atención sanitaria.

\section{Referencias bibliográficas}

1. Baixauli Fernández VJ, Satué de Velasco E, Gil García MI, Roig Sena JC, Villasuso Cores B, Sáenz de Buruaga Pérez de Atxa S. Propuesta de la Sociedad Española de Farmacia Comunitaria (SEFAC) sobre servicios profesionales farmacéuticos en farmacia comunitaria. Farmacéuticos Comunitarios 2013; 5(3): 119-126.

2. Mancia G, Fagard R, Narkiewicz K, Redón J, Zanchetti A, Böhm M, et al. Task Force Members. 2013 ESH/ ESC Guidelines for the management of arterial hypertension: the Task Force for the management of arterial hypertension of the European Society of Hypertension (ESH) and of the European Society of Cardiology (ESC). J Hypertens. 2013; 31:1281-1357. doi:10.1097/01. hjh.0000431740.32696.cc

3. Quinto Grupo de Trabajo de la Sociedad Europea de Cardiología y otras Sociedades sobre la Prevención de la Enfermedad Cardiovascular en la Práctica Clínica. Guía europea sobre prevención de la enfermedad cardiovascular en la práctica clínica. Rev Esp Cardiol. 2012; 65(10):937. e1-e66.

4. Mahmood SS, Levy D, Vasan RS, Wang TJ. The Framingham Heart Study and the epidemiology of cardiovascular disease: a historical perspective. Lancet. 2014 Mar 15; 383(9921):999-1008. doi:10.1016/ S0140-6736(13)61752-3

5. Conroy RM, Pyorala K, Fitzgerald AP, Sans S, Menotti A, DeBacker G, et al. Estimation of ten year risk of fatal cardiovascular disease in Europe: the SCORE project. Eur Heart J 2003; 24:987-1003. doi:10.1016/S0195668X(03)00114-3

6. Foraster M, Basagaña X, Aguilera I, Rivera M, Agis D, Bouso L, et al. As- sociation of long-term exposure to traffic-related air pollution with blood pressure and hypertension in an adult population-based cohort in Spain (the REGICOR study). Environ Health Perspect 2014 Apr; 122(4):404-411. doi:10.1289/ehp.1306497

7. Marrugat J, Vila J, Baena Díez JM, Grau M, Sala J, Ramos R, et al. Validez relativa de la estimación del riesgo cardiovascular a 10 años en una cohorte poblacional del estudio REGICOR. Rev Esp Cardiol 2011 May; 64(5):385-94. doi:10.1016/j.recesp.2010.12.011

8. Royo Bordonada MA, Lobos Bejarano JM, Villar Álvarez F, Sans S, Pérez A, Pedro Botet J, et al. Comentarios del comité español interdisciplinario de prevención cardiovascular (CEIPC) a las guías europeas de prevención cardiovascular 2012. Rev Esp Salud Pública. 2013; 87:103-120.

9. Bofí Martínez P, García Jiménez E, Martínez Martínez F. Comparación de las intervenciones de educación sanitaria y de seguimiento farmacoterapéutico en pacientes con factores de riesgo cardiovascular que acuden a una farmacia comunitaria (Estudio FISFTES-PM). Aten Primaria 2015; 47(3):141-148. doi:10.1016/j. aprim.2014.04.012

10. Rodríguez Chamorro A, García Jiménez E, Rodríguez Chamorro MA, Amariles P, Martínez Martínez F, Pérez Merino E, et al. Effectiveness of Pharmacotherapy Follow-Up for the Control of Hypertensive Patients in Community Pharmacies: EMDADER-HTA Study. Latin American Journal of Pharmacy. 2013; 32 (7): 982-8.

11. Amariles $P$, Sabater Hernández D, García-Jiménez E, Rodríguez Chamorro MÁ, Prats-Más R, Marín Magán F, et al. Effectiveness of Dader Method for pharmaceutical care on control of blood pressure and total cholesterol in outpatients with cardiovascular disease or cardiovascular risk: EMDADER-CV randomized controlled trial. J Manag Care Pharm. 2012 May; 18(4): 311-23. doi:10.18553/ jmcp.2012.18.4.311

12. Chamorro MA, García Jiménez E, Amariles P, Chamorro AR, Merino EM, Martínez FM, et al. Efecto de la actuación farmacéutica en la adherencia del tratamiento farmacológico de pacientes ambulatorios con riesgo cardiovascular (Estudio EMDADER-CV-INCUMPLIMIENTO). Aten Primaria. 2011 May; 43(5): 245-53. doi:10.1016/j.aprim.2010.05.006 